\title{
AN UNCERTAINTY PRINCIPLE ON HYPERBOLIC SPACE
}

\author{
LIMIN SUN
}

(Communicated by J. Marshall Ash)

\begin{abstract}
In this paper, we establish an uncertainty principle on hyperbolic space $H^{n}=S O_{e}(n, 1) / S O(n)$, which prohibits $f$ from being confined to small neighborhoods around any point in $H^{n}$ under certain assumptions on the Fourier transform $\tilde{f}$, where $f$ is a normalized $L^{2}$ function on $H^{n}$.
\end{abstract}

\section{INTRODUCTION}

For a function $f \in L^{2}\left(R^{n}\right)$ with $\|f\|_{2}=1$, let $\hat{f}$ be its Fourier transform. The classical Heisenberg uncertainty principle asserts that the product of the variances of the probability measures $|f(x)|^{2} d x$ and $|\hat{f}(x)|^{2} d x$ is larger than an absolute constant. Recently, Strichartz [3] gave a new generalization of the Heisenberg uncertainty principle, and posed a question: what conditions on the Fourier transform of a normalized $L^{2}$ function will force the variance of $|f(x)|^{2} d x$ to be large? As the author stated in his paper, this question makes sense in any context in which some sort of Fourier transform is defined. On the other hand, the Fourier transform on hyperbolic space $H^{n}=S O_{e}(n, 1) / S O(n)$ can be defined following Helgason [1]. This naturally leads us to consider the above question on $H^{n}$ in the present paper.

\section{Preliminaries}

Throughout this paper, we use ch, sh, and th as the simplified notations for cosh, sinh, and tanh respectively.

Let $G=S O_{e}(n, 1)$ be the identity component of the group $S O(n, 1)=$ $\left\{g \in G L(n+1, R) ; g I_{n, 1} g^{t}=I_{n, 1}\right\}$, where $I_{n, 1}=\operatorname{diag}(1, \ldots, 1,-1) \in$ $G L(n+1, R)$. The group $K=S O(n)$ is naturally imbedded in $S O_{e}(n, 1)$. It is well known that $X=S O_{e}(n, 1) / S O(n)$ is a Riemannian symmetric space which is usually denoted by $H^{n}$ and called hyperbolic space.

For further discussions, we take the open unit ball as a model for $H^{n}$, i.e., $X=\left\{x=\left(x_{1}, \ldots, x_{n}\right) \in R^{n} ;|x|<1\right\}$ with Riemannian structure $d s^{2}=$ $4\left(1-|x|^{2}\right)^{-2}\left(d x_{1}^{2}+\cdots+d x_{n}^{2}\right)$, where $|\cdot|$ denotes the Euclidean norm in $R^{n}$.

Received by the editors September 10, 1991 and, in revised form, September 15, 1992.

1991 Mathematics Subject Classification. Primary 43A32, 43A85.

Key words and phrases. Hyperbolic space, uncertainty principle.

This project was supported by the National Natural Science Foundation of China, NSFC 19301016. 
Then the $G$-invariant measure on $X$ is given by

$$
d x=\left(2\left(1-|x|^{2}\right)^{-1}\right)^{n} d x_{1} \cdots d x_{n},
$$

where $d x_{1} \cdots d x_{n}$ is the Lebesgue measure on $R^{n}$; and the $K$-invariant measure $d b$ on the boundary $B \quad\left(=S^{n-1}\right)$ is the standard surface element on $S^{n-1}$. The geodesic distance from $x \in X$ to the origin $o \in X$ is given by

$$
d(o, x)=\log \frac{1+|x|}{1-|x|}, \quad \text { i.e., } \operatorname{th}\left(\frac{d(o, x)}{2}\right)=|x| .
$$

Let $\langle x, b\rangle$ be the (signed) distance from $o$ to the horosphere passing through $x \in X$ and $b \in B$. A simple geometric observation shows that

$$
e^{\langle x, b\rangle}=\left(1-|x|^{2}\right) /|x-b|^{2} \text {. }
$$

The Fourier transform $\tilde{f}$ of a function $f \in C_{0}^{\infty}(X)$ is defined by

$$
\tilde{f}(\lambda, b)=\int_{X} e^{(-i \lambda+(n-1) / 2)\langle x, b\rangle} f(x) d x \text { for } \lambda \in C \text { and } b \in B .
$$

According to the general result by Helgason (cf. [2]), we have

Theorem A. The mapping $f \rightarrow \tilde{f}$ extends to an isometry from $L^{2}(X, d x)$ onto $L^{2}\left(R_{+} \times B,|C(r)|^{-2} d r d b\right)$. Here, $C(\cdot)$ is Harish-Chandra's c-function.

\section{Statement of the Result}

Let $\left\{r_{k}\right\}$ be a series of real numbers satisfying the following conditions:

$$
d_{0} / 2 \leq r_{1} \leq d_{0} \leq 1, \quad 0 \leq r_{k+1}-r_{k} \leq d_{0}, \quad k=1,2, \ldots,
$$

Set

$$
E=\bigcup_{k=1}^{\infty}\left\{(r, b) \in R_{+} \times B ; r=r_{k}\right\}
$$

A measure $m(r, b) d r d b$ on $R_{+} \times B$ can be induced on the submanifold $E$ in the following manner:

$$
\int_{E} h(r, b) m(r, b) d r d b=\sum_{k=1}^{\infty} \int_{E} h\left(r_{k}, b\right) m\left(r_{k}, b\right) d b .
$$

The main result of this paper is as follows.

Theorem 1. Let $r_{k}(k=1,2, \ldots)$ and $E$ be given as in (3) and (4) respectively. Suppose that $f \in L^{2}(X, d x)$ with $\|f\|_{2}=1$. Fix an $\varepsilon \in(0,1)$. Then for any sufficiently small $c>0$, there exists a constant $c^{\prime}$ such that

$$
\inf _{y \in X} \max \left(\int_{X} d(x, y)^{2}|f(x)|^{2} d x, \int_{X} d(x, y)^{2+2 \varepsilon}|f(x)|^{2} d x\right) \geq c^{\prime} d_{0}^{-2}
$$

provided that

$$
\int_{E}|\tilde{f}(r, b)|^{2} \cdot|C(r)|^{2} d r d b \leq c \cdot d_{0}^{-1} .
$$


Remark 1. Actually, we can choose $c^{\prime}=\left(2\left(1-\sqrt{3^{n} C^{*} c}\right)\right)^{2} /\left(3^{n+2} C^{*} C(\varepsilon)\right)$ for any $0<c<\left(3^{n} C^{*}\right)^{-1}$, where $C^{*}=\operatorname{ch}^{2}(2 \pi) \cdot(n+1)^{n-2}$ and $C(\varepsilon)=$ $2(1+16 / \varepsilon)^{2}$.

\section{SOME LEMMAS}

For our purpose, we need an asymptotical estimation about the HarishChandra function.

Proposition 1. Let $C(r)$ be the Harish-Chandra function in Theorem A. Then there exist constants $\alpha$ and $\beta$ dependent only on the dimension $n$ such that $\alpha r^{h-1} \leq|C(r)|^{-2} \leq \beta r^{n-1}$ for $r \geq 1$, and $\alpha r^{2} \leq|C(r)|^{-2} \leq \beta r^{2}$ for $0<r \leq 2$.

Proof. We know from [5] that there exists a constant $C_{n}$ dependent only on $n$ such that, for $r \in R$,

$$
|C(r)|^{-2}=C_{n}\left[r^{2}+\left(\frac{1}{2}\right)^{2}\right] \cdots\left[r^{2}+\left(\frac{2 m-3}{2}\right)^{2}\right] r \cdot \operatorname{th}(\pi r), \quad n=2 m,
$$

and

$$
|C(r)|^{-2}=C_{n} \cdot r^{2}\left(r^{2}+1\right) \cdots\left(r^{2}+(m-1)^{2}\right), \quad n=2 m+1 .
$$

Hence, the conclusion follows.

Remark 2. In fact, one can choose $\alpha=\pi C_{n}\left(\operatorname{ch}^{2}(2 \pi) 2^{n-2}\right)^{-1}$ and $\beta=$ $\pi C_{n}\left(4+(n-3)^{2} / 4\right)^{(n-2) / 2}$. Note that $\beta / \alpha \leq C^{*}$.

Lemma 1. Let $\left\{r_{k}\right\}$ be given as in (3). If $m_{0}=0$ and $m_{k}=\frac{1}{2}\left(r_{k}+r_{k+1}\right)$ for $k \geq 1$, then

$$
\left|C\left(r_{k}\right)\right|^{2} \int_{m_{k-1}}^{m_{k}}|C(r)|^{-2} d r \leq\left(3^{n} C^{*}\right) d_{0}
$$

Proof. (I) The case $k \geq 2$. By the mean value theorem about integration, there exists $t_{k} \in\left(m_{k-1}, m_{k}\right)$ such that

$$
\left|C\left(r_{k}\right)\right|^{2} \int_{m_{k-1}}^{m_{k}}|C(r)|^{-2} d r=\left(m_{k}-m_{k-1}\right)\left|C\left(r_{k}\right) / C\left(t_{k}\right)\right|^{2} \text {. }
$$

But, $m_{k}-m_{k-1}=\left(r_{k+1}-r_{k}\right) / 2+\left(r_{k}-r_{k-1}\right) / 2 \leq d_{0} \leq 1$, and $m_{k-1} \geq r_{1} \geq d_{0} / 2$ for $k \geq 2$. Hence (note that $r_{k}, t_{k} \in\left(m_{k-1}, m_{k}\right)$ ),

$$
\left|r_{k} / t_{k}\right| \leq m_{k} / m_{k-1}=\left[1+\left(m_{k}-m_{k-1}\right) / m_{k-1}\right] \leq 3 \text {. }
$$

Moreover, $r_{k}, t_{k} \geq 1$ if $m_{k-1} \geq 1$, and $r_{k}, t_{k}<2$ if $m_{k-1}<1$. Thus, one gets from (5), (6), and Proposition 1 that

and

$$
\left|C\left(r_{k}\right)\right|^{2} \int_{m_{k-1}}^{m_{k}}|C(r)|^{-2} d r \leq C^{*}\left|\frac{r_{k}}{t_{k}}\right|^{n-1} d_{0} \leq\left(3^{n-1} C^{*}\right) d_{0} \quad \text { if } m_{k-1} \geq 1,
$$

$$
\left|C\left(r_{k}\right)\right|^{2} \int_{m_{k-1}}^{m_{k}}|C(r)|^{-2} d r \leq C^{*}\left|\frac{r_{k}}{t_{k}}\right|^{n-1} d_{0} \leq\left(9 C^{*}\right) d_{0} \quad \text { if } m_{k-1}<1 .
$$

(II) The case $k=1$. Since $m_{0}=0$ and $d_{0} / 2 \leq r_{1} \leq m_{1} \leq 3 d_{0} / 2$. Applying Proposition 1, a simple calculation shows

$$
\begin{aligned}
\left|C\left(r_{1}\right)\right|^{2} \int_{0}^{m_{1}}|C(r)|^{-2} d r & \leq C^{*} r_{1}^{-2} \int_{0}^{m_{1}} r^{2} d r \\
& =C^{*}\left(m_{1} / r_{1}\right)^{2}\left(m_{1} / 3\right)<\left(9 C^{*}\right) d_{0} .
\end{aligned}
$$

The proof of Lemma 1 is complete. 
Lemma 2. Let $\left\{r_{k}\right\},\left\{m_{k}\right\}$ be given as in Lemma 1. Write

$$
a_{k}=\int_{r_{k}}^{m_{k}} \int_{r_{k}}^{m_{k}}\left|\frac{C(r)}{C(t)}\right|^{2} d t d r, \quad b_{k}=\int_{m_{k-1}}^{r_{k}} \int_{m_{k-1}}^{r_{k}}\left|\frac{C(r)}{C(t)}\right|^{2} d t d r .
$$

Then $a_{k} \leq\left(3^{n} C^{*} / 4\right) d_{0}^{2}$ for $k \geq 1$, and $b_{k} \leq\left(3^{n} C^{*} / 4\right) d_{0}^{2}$ for $k \geq 2$.

Proof. Again by the mean value theorem, there exist $t_{k}$ and $s_{k} \in\left(r_{k}, m_{k}\right)$ such that

$$
a_{k}=\left(m_{k}-r_{k}\right)^{2}\left|C\left(s_{k}\right) / C\left(t_{k}\right)\right|^{2} \leq\left(d_{0} / 2\right)^{2}\left|C\left(s_{k}\right) / C\left(t_{k}\right)\right|^{2} .
$$

The remaining arguments are the same as those used in the proof of Lemma 1 (the case $k \geq 2$ ). Since $m_{0}$ is not involved, we can deal with $b_{k}$ in a similar way.

Lemma 3. Let $\left\{r_{k}\right\},\left\{m_{k}\right\}$ be given as in Lemma 1 . If $\tilde{f}$ is the Fourier transform of a function $f$ on $X$, then for $k \geq 1$,

$$
\begin{aligned}
\int_{m_{k-1}}^{m_{k}} & \left(\int_{r_{k}}^{r}\left(\frac{\partial \tilde{f}}{\partial s}\right)(s, b) d s\right)^{2}|C(r)|^{-2} d r \\
\quad \leq & \left(\frac{3^{n} C^{*}}{4}\right) d_{0}^{2} \int_{m_{k-1}}^{m_{k}}\left|\left(\frac{\partial \tilde{f}}{\partial s}\right)(s, b)\right|^{2}|C(s)|^{-2} d s .
\end{aligned}
$$

Proof. For convenience, write $d u(r)=|C(r)|^{-2} d r$. The Hölder inequality simply yields

$$
\left(\int_{r_{k}}^{r}\left(\frac{\partial \tilde{f}}{\partial r}\right)(s, b) d s\right)^{2} \leq p\left(r, r_{k}\right) \int_{r_{k}}^{r}\left|\left(\frac{\partial \tilde{f}}{\partial r}\right)(s, b)\right|^{2} d u(s),
$$

where $p\left(r, r_{k}\right)=\int_{r_{k}}^{r}|C(s)|^{2} d s$. Changing the order of the integration, we get

$$
\begin{aligned}
\int_{m_{k-1}}^{m_{k}} & \left(\int_{r_{k}}^{r}\left(\frac{\partial \tilde{f}}{\partial r}\right)(s, b) d s\right)^{2}|C(s)|^{-2} d s \\
\leq & \int_{m_{k-1}}^{r_{k}}\left|\left(\frac{\partial \tilde{f}}{\partial r}\right)(s, b)\right|^{2} d u(s) \int_{m_{k-1}}^{s}-p\left(r, r_{k}\right) d u(r) \\
& \quad+\int_{r_{k}}^{m_{k}}\left|\left(\frac{\partial \tilde{f}}{\partial r}\right)(s, b)\right|^{2} d u(s) \int_{s}^{m_{k}} p\left(r, r_{k}\right) d u(r) .
\end{aligned}
$$

However, it follows from Lemma 2 that, for $k \geq 1$ and $r_{k} \leq s \leq m_{k}$,

$$
\int_{s}^{m_{k}} p\left(r, r_{k}\right) d u(r) \leq \int_{r_{k}}^{m_{k}} \int_{r_{k}}^{m_{k}}\left|\frac{C(s)}{C(r)}\right|^{2} d s d r=a_{k} \leq\left(\frac{3^{n} C^{*}}{4}\right) d_{0}^{2} .
$$

Similary, for $k \geq 2$ and $m_{k-1} \leq s \leq r_{k}$,

$$
\int_{m_{k-1}}^{s}-p\left(r, r_{k}\right) d u(r) \leq b_{k} \leq\left(\frac{3^{n} C^{*}}{4}\right) d_{0}^{2} .
$$


Finally, for $0<r \leq r_{1} \leq 1$,

$$
-p\left(r, r_{1}\right)=\int_{r}^{r_{1}}|C(t)|^{2} d t \leq \alpha^{-1} \int_{r}^{r_{1}} t^{-2} d t \leq(\alpha r)^{-1},
$$

and hence, for $0<s \leq r_{1} \leq d_{0}$,

$$
\int_{0}^{s}-p\left(r, r_{1}\right) d u(r) \leq \frac{\beta}{\alpha} \int_{0}^{s} r \cdot d r \leq\left(\frac{C^{*}}{2}\right) d_{0}^{2} .
$$

Now, Lemma 3 can be derived from (7)-(10) immediately.

Let $R(x)=\log \left[\left(1+|x|^{2}\right) /\left(1-|x|^{2}\right)\right]$. Then,

$$
R(x) \leq \log \left[(1+|x|)^{2} /\left(1-|x|^{2}\right)\right]=d(o, x),
$$

and

$$
\log \left(1-|x|^{2}\right) /|x-b|^{2}=-\left\{R(x)+\log \left[|x-b|^{2} /\left(1+|x|^{2}\right)\right]\right\} .
$$

For $b=\left(b_{1}, \ldots, b_{n}\right) \in S^{n-1}$ and $l=\left(l_{1}, \ldots, l_{n}\right) \in Z_{+}^{n}$, let $A_{l}(b)=\left(b_{1}\right)^{l_{1}}$ $\cdots\left(b_{n}\right)^{l_{n}}$ and $|l|=l_{1}+\cdots+l_{n}$. By Taylor's expansion,

$$
\log \frac{|x-b|^{2}}{1+|x|^{2}}=\log \left[1-\frac{2 x \cdot b}{1+|x|^{2}}\right]=\sum_{k=1}^{\infty} \frac{1}{k} \sum_{|l|=k} C_{k}^{l} A_{l}(b) Q_{k, l}(x),
$$

where $C_{k}^{l}=k ! /\left(l_{1} !\right) \cdots\left(l_{n} !\right)$ and $Q_{k, l}(x)=\left(2|x| /\left(1+|x|^{2}\right)\right)^{k} A_{l}\left(x^{\prime}\right), x^{\prime}=x /|x|$.

Lemma 4. Let $\tilde{f}$ be the Fourier transform of a function $f$ on $X$. Then, for any $0<\varepsilon<1$,

$$
\begin{aligned}
& \int_{R_{+} \times B}\left|\left(\frac{\partial \tilde{f}}{\partial r}\right)(r, b)\right|^{2} \cdot|C(r)|^{-2} d r d b \\
& \leq C(\varepsilon) \max \left(\int_{X} d(o, x)^{2}|f(x)|^{2} d x, \int_{X} d(o, x)^{2+2 \varepsilon}|f(x)|^{2} d x\right) .
\end{aligned}
$$

Proof. A direct calculation together with (12) and (13) shows

$$
\begin{aligned}
\left(\frac{\partial \tilde{f}}{\partial r}\right)(r, b) & =-i \int_{X}\left(\log \frac{1-|x|^{2}}{|x-b|^{2}}\right) f(x)\left(\frac{1-|x|^{2}}{|x-b|^{2}}\right)^{i r+(n-1) / 2} d x \\
& =-i(\widetilde{R f})(r, b)-i \sum_{k=1}^{\infty} \frac{1}{k} \sum_{|l|=k} C_{k}^{l} A_{l}(b)\left(\widetilde{Q_{k, l}} f\right)(r, b) .
\end{aligned}
$$

But, $\sum_{|l|=k} C_{k}^{l} A_{l}(b)^{2}=\left(b_{1}^{2}+\cdots+b_{n}^{2}\right)^{k}=1$. Hence, by Hölder's inequality we get that, for any $0<\varepsilon<1$,

$$
\left(\frac{\partial \tilde{f}}{\partial r}\right)^{2} \leq 2\left\{\left.\widetilde{R f}\right|^{2}+C_{1}(\varepsilon) \sum_{k=1}^{\infty} \frac{(1+\log k)^{1+\varepsilon}}{k} \sum_{|l|=k} C_{k}^{l}\left|\widetilde{Q_{k, l}} f\right|^{2}\right\}
$$

where $C_{1}(\varepsilon)=\sum_{k=1}^{\infty} 1 / k(1+\log k)^{1+\varepsilon}$. This fact together with Theorem A 
shows that

$$
\begin{aligned}
\int_{R_{+} \times B} \mid & \left.\mid \frac{\partial \tilde{f}}{\partial r}\right)\left.(r, b)\right|^{2} \cdot|C(r)|^{-2} d r d b \\
\leq 2 & \left\{\int_{X} d(o, x)^{2}|f(x)|^{2} d x\right. \\
& \left.+C_{1}(\varepsilon) \int_{X} \sum_{k=1}^{\infty} \frac{(1+\log k)^{1+\varepsilon}}{k} \sum_{|l|=k} C_{k}^{l}\left|Q_{k, l}(x)\right|^{2}|f(x)|^{2} d x\right\} .
\end{aligned}
$$

However, a simple calculation yields that

$$
\begin{gathered}
\sum_{k=1}^{\infty} \frac{(1+\log k)^{1+\varepsilon}}{k} \sum_{|l|=k} C_{k}^{l}\left|Q_{k, l}(x)\right|^{2}=\sum_{k=1}^{\infty} \frac{(1+\log k)^{1+\varepsilon}}{k} \operatorname{th}(d(o, x))^{2 k} \\
\leq d(o, x)^{2}+\sum_{k \geq 2} \frac{(1+\log k)^{1+\varepsilon}}{k} M_{k, \varepsilon}^{2} d(o, x)^{2+2 \varepsilon},
\end{gathered}
$$

where $M_{k, \varepsilon}=\max _{r>0}\left\{\operatorname{th}(r)^{k} / r^{1+\varepsilon}\right\}$. Obviously, $M_{k, \varepsilon} \leq 1$ for all $k \geq 2$. If $r_{k, \varepsilon}$ is a stable point of $\operatorname{th}(r)^{k} / r^{1+\varepsilon}$ then $r_{k, \varepsilon}$ must satisfy the equation $2 r k=(1+\varepsilon) \operatorname{ch}(2 r)$. Hence, $r_{k, \varepsilon} \geq \frac{1}{2} \log (k-1)$ and $M_{k, \varepsilon} \leq[2 / \log (k-1)]^{1+\varepsilon}$ for $k \geq 4$. Finally, we get

$$
\begin{aligned}
\int_{R_{+} \times B} \mid & \left|\left(\frac{\partial \tilde{f}}{\partial r}\right)(r, b)\right|^{2}|C(r)|^{-2} d r d b \\
\leq & 2\left(1+C_{1}(\varepsilon)+C_{1}(\varepsilon) C_{2}(\varepsilon)\right) \\
& \times \max \left(\int_{X} d(o, x)^{2}|f(x)|^{2} d x, \int_{X} d(o, x)^{2+2 \varepsilon}|f(x)|^{2} d x\right),
\end{aligned}
$$

where $C_{2}(\varepsilon)=6+4^{1+\varepsilon} \sum_{k>4}(1+\log k)^{1+\varepsilon} / k(\log (k-1))^{2+2 \varepsilon}$. It is easy to check that $C_{1}(\varepsilon)<2 / \varepsilon$ and $C_{2}(\varepsilon) \leq 6+2^{7} / \varepsilon$. This completes the proof.

\section{PROOF OF THE THEOREM}

First, we note the following fact that can be derived from the formulas in [1, pp. 197, 418].

Proposition 2. For a function $f$ on $X$, let $f_{g}(x)=f(g x), g \in S O_{\varepsilon}(n, 1)$. Then

$$
\tilde{f}_{g}(\lambda, b)=e^{(i \lambda+(n-1) / 2)\langle g o, g b\rangle} \tilde{f}(\lambda, g b),
$$

$$
\int_{B}\left|\tilde{f}_{g}(\lambda, b)\right|^{2} d b=\int_{B}|\tilde{f}(\lambda, b)|^{2} d b .
$$

Now, we begin to prove Theorem 1. Suppose that $f$ is a function on $X$ such that $\int_{X}(d(o, x))^{2+2 \varepsilon}|f(x)|^{2} d x<\infty$ (if not, there is nothing to prove). Note the identity:

$$
\tilde{f}(r, b)=\tilde{f}\left(r_{k}, b\right)+\int_{r_{k}}^{r}\left(\frac{\partial \tilde{f}}{\partial r}\right)(s, b) d s .
$$


An application of Minkowski's inequality together with Lemmas 1 and 2 yields

$$
\begin{aligned}
& \left(\int_{m_{k-1}}^{m_{k}}|\tilde{f}(r, b)|^{2}|C(r)|^{-2} d r\right)^{1 / 2} \\
& \leq\left|\tilde{f}\left(r_{k}, b\right)\right|\left|C\left(r_{k}\right)\right|^{-1}+\left(\left|C\left(r_{k}\right)\right|^{2} \int_{m_{k-1}}^{m_{k}}|C(r)|^{-2} d r\right)^{1 / 2} \\
& \quad+\left(\int_{m_{k-1}}^{m_{k}}\left(\int_{r_{k}}^{r}\left(\frac{\partial \tilde{f}}{\partial r}\right)(s, b) d s\right)^{2}|C(r)|^{-2} d r\right)^{1 / 2} \\
& \leq C_{1} \cdot d_{0}^{1 / 2}\left|\tilde{f}\left(r_{k} b\right)\right|\left|C\left(r_{k}\right)\right|^{-1} \\
& \quad+C_{2} \cdot d_{0}\left(\int_{m_{k-1}}^{m_{k}}\left|\left(\frac{\partial \tilde{f}}{\partial r}\right)(s, b)\right|^{2}|C(s)|^{-2} d s\right)^{1 / 2}
\end{aligned}
$$

where $C_{1}=2 C_{2}=\left(3^{n} C^{*}\right)^{1 / 2}$. Another application of Minkowski's inequality together with Theorem $A$ and Lemma 4 shows that

$$
\begin{aligned}
& 1=\left(\int_{R_{+} \times B}|\tilde{f}(r, b)|^{2}|C(r)|^{-2} d r d b\right)^{1 / 2} \\
& \leq C_{1} d_{0}^{1 / 2}\left(\int_{B} \sum_{k=1}^{\infty}\left|\tilde{f}\left(r_{k}, b\right)\right|^{2}\left|C\left(r_{k}\right)\right|^{-2} d b\right)^{1 / 2} \\
&+C_{2} d_{0}\left(\int_{R_{+} \times B}\left|\left(\frac{\partial \tilde{f}}{\partial r}\right)(s, b)\right|^{2}|C(s)|^{-2} d s d b\right)^{1 / 2} \\
& \leq C_{1} d_{0}^{1 / 2}\left(\int_{E}|\tilde{f}(r, b)|^{2}|C(r)|^{-2} d r d b\right)^{1 / 2} \\
&+C_{2} C(\varepsilon)^{1 / 2} d_{0} \max \left\{\left(\int_{X} d(o, x)^{2}|f(x)|^{2} d x\right)^{1 / 2},\right. \\
&\left.\left(\int_{X} d(o, x)^{2+2 \varepsilon}|f(x)|^{2} d x\right)^{1 / 2}\right\}
\end{aligned}
$$

Any $y \in X$ can be written as $y=g o, g \in S O_{e}(n, 1)$. Proposition 2 implies that (14) remains true when $d(o, x)$ is replaced by $d(x, y)$. Hence, Theorem 1 is a direct consequence of (14).

Let $I(X)$ be the space consisting of radial functions on $X$. For $f \in I(X)$, one can define its spherical transform by

$$
\tilde{f}(\lambda)=\int_{X} f(x) \phi_{-\lambda}(x) d x, \quad \phi_{\lambda}(x)=\int_{B} e^{(i \lambda+(n-1) / 2)\langle x, b\rangle} d b .
$$

Note that $\tilde{f}(\lambda, b)=\tilde{f}(\lambda)$ for $f \in I(X)$, and $\int_{B} d b=1$. Consequently,

Theorem 2. Let $\left\{r_{k}\right\}$ be given as in (3) and $r_{-k}=-r_{k}, k=1,2, \ldots$ Suppose that $f \in I(X) \cap L^{2}(X)$ with $\|f\|_{2}=1$. Fix an $\varepsilon \in(0,1)$. Then for any 
sufficiently small $c>0$, there exists constant $c^{\prime}$ such that

$$
\inf _{y \in X} \max \left(\int_{X} d(x, y)^{2}|f(x)|^{2} d x, \int_{X} d(x, y)^{2+2 \varepsilon}|f(x)|^{2} d x\right) \geq c^{\prime} d_{0}^{-2}
$$

provided that $\sum_{k=-\infty}^{+\infty}\left|C\left(r_{k}\right)\right|^{-2}\left|\tilde{f}\left(r_{k}\right)\right|^{2} \leq c \cdot d_{0}^{-1}$.

\section{A FURTHER RESULT}

Obviously, for a radial function $f$ on $X$, one should study its confinement to some annular neighborhood $N_{\delta}(R)=\{x \in X ;|d(o, x)-R|<\delta\}$; i.e., we hope to get the lower bound for

$$
\int_{X}|d(o, x)-R|^{2}|f(x)|^{2} d x, \quad R>0,
$$

under suitable conditions on the Fourier transform of $f$. Here, we present an example to explain this point. and

$$
\begin{aligned}
\phi_{-\lambda}(x) & =2 \pi \int_{0}^{\pi}(\operatorname{ch} r-\operatorname{sh} r \cdot \cos \theta)^{-1+i \lambda}(\sin \theta)^{n-2} d \theta \\
& =-4 \pi \sin (\lambda r) / \lambda \operatorname{sh}(r) \text { for } d(o, x)=r .
\end{aligned}
$$

Calculating under the geodesic coordinates, we have $d x=(\operatorname{sh} r)^{2} d r d w$, where $d w$ is the standard surface element on $S^{2}$. Hence, each radial function $f(x) \in$ $I(X) \cap L^{2}(X, d x)$ corresponds to a function $F_{f}(r) \in L_{\text {odd }}^{2}(R, d r)$ such that $f(x)=F_{f}(r) / \operatorname{sh}(r)$ for $r=d(o, x)$, where $L_{\text {odd }}^{2}(R, d r)$ denotes the space consisting of odd functions which are square integrable on $R$.

Proposition 3. The mapping: $f \rightarrow F_{f}$ is an isometry from $I(X) \cap L^{2}(X, d x)$ onto $L_{\text {odd }}^{2}(R, d r / 2)$. Moreover, $\tilde{f}(\lambda)=-i(2 \pi)^{-1 / 2}|C(\lambda)| \cdot \widehat{F}_{f}(\lambda)$ for $\lambda>0$, where $\tilde{f}$ is the spherical transform of $f$ and $\widehat{F}_{f}$ is the Euclidean Fourier transform of $F_{f}$.

Proof. The first assertion is obvious. In the present case, $|C(\lambda)|^{-2}=(2 \pi)^{-3} \lambda^{2}$ for $\lambda>0$. By (15) a direct calculation shows

$$
\begin{aligned}
\tilde{f}(\lambda) & =\int_{X} f(x) \phi_{-\lambda}(x) d x=-i\left(\frac{2 \pi}{\lambda}\right) \int_{-\infty}^{+\infty} F_{f}(r) e^{-i \lambda r} d r \\
& =i(2 \pi)^{-1 / 2}|C(\lambda)| \widehat{F}_{f}(\lambda), \quad \lambda>0 .
\end{aligned}
$$

Theorem 3. Let $\left\{r_{k}\right\}$ be given as in (3). Suppose that $f \in I(X) \cap L^{2}(X, d x)$ with $\|f\|_{2}=1$. Then for any $0<\delta<1$,

$$
\inf _{y \in X, R \geq 0} \int_{X}|d(x, y)-R|^{2}|f(x)|^{2} d x \geq \frac{8 \delta^{2}}{d_{0}^{2}},
$$

provided that $\sum_{k=1}^{\infty}\left|\tilde{f}\left(r_{k}\right)\right|^{2}\left|C\left(r_{k}\right)\right|^{-2} \leq\left(1-\delta^{2}\right) / 2 \pi d_{0}$. Here, $X$ is three dimensional hyperbolic space.

Proof. Since the measure $d x$ is invariant, (16) is equivalent to

$$
\inf _{R \geq 0} \int_{X}|d(o, x)-R|^{2}|f(x)|^{2} d x \geq \frac{8 \delta^{2}}{d_{0}^{2}}
$$


By Proposition 3, we need only show

$$
\inf _{R \geq 0} \frac{1}{2} \int_{-\infty}^{\infty}|r-R|^{2}\left|F_{f}(r)\right|^{2} d r \geq \frac{8 \delta^{2}}{d_{0}^{2}}
$$

Again by Proposition 3, one sees that

$$
\begin{aligned}
\left(\frac{1}{2} \sum_{k=-\infty}^{\infty}\left|\widehat{F}_{f}\left(r_{k}\right)\right|^{2}\right)^{1 / 2} & =\left(\sum_{k=1}^{\infty}\left|\widehat{F}_{f}\left(r_{k}\right)\right|^{2}\right)^{1 / 2} \\
& =\left(2 \pi \sum_{k=1}^{\infty}\left|\tilde{f}\left(r_{k}\right)\right|^{2}\left|C\left(r_{k}\right)\right|^{-2}\right)^{1 / 2} \leq \frac{1-\delta}{d_{0}^{1 / 2}}
\end{aligned}
$$

The conclusion follows from the result by Strichartz in [3, p. 98].

Remark 3. It would be interesting to know whether the conclusion in Theorem 3 is valid in general hyperbolic space. To deal with this problem will require a new technique since the one we used relies on the particular expression of the spherical function.

\section{ACKNOWLEDGMENT}

The author would like to thank Professor R. S. Strichartz for his generous comments and suggestions on the original manuscript, which made this work possible.

\section{REFERENCES}

1. S. Helgason, Groups and geometric analysis: integral geometry, invariant differential operators, and spherical functions, Pure and Appl. Math., vol. 113, Academic Press, Orlando, 1984.

2. _ Paley-Wiener theorems and surjectivity of invariant differential operatores on symmetric spaces and Lie groups, Bull. Amer. Math. Soc. 79 (1973), 129-132.

3. R. S. Strichartz, Uncertainty principles in harmonic analysis, J. Funct. Anal. 84 (1989), 97-114.

4. - Harmonic analysis as spectral theory of Laplacians, J. Funct. Anal. 87 (1989), 51-148.

5. R. Takahashi, Sur les représentations unitaires des groupe de Lorentz généralisés, Bull. Soc. Math. France 91 (1963), 289-433.

Department of Mathematics, Hangzhou University, Hangzhou, Zhejiang, 310028, Peoples Republic of China 